\title{
Methodology to Find and Mitigate Transient Recovery Voltages (T.R.V) Considering Various Abnormal Scenarios: A Case Study of IEEE-39 Bus System
}

\author{
Rahman Ullah Khan ${ }^{1}$, Dr. Abdul Basit ${ }^{2}$ \\ ${ }^{1,2}$ US-Pakistan Center for Advanced Studies in-Energy (USPCAS-E), University of Engineering \& Technology \\ (UET) Peshawar, Pakistan. \\ engr.rk@live.com ${ }^{1}$, abdul.basit@uetpeshawar.edu.pk ${ }^{2}$ \\ Received: 18 December, Revised: 26 December, Accepted: 27 December
}

\begin{abstract}
Abnormal switching transients are those transients that arise during unbalanced faults interruption e.g., single-lineto-ground (S.L.G) fault, double-line-to-ground (D.L.G) fault, or three-phase fault. Circuit-breaker is the first line of defense to face these abnormal transients. Whenever a circuit-breaker encounters these abnormal transients, it changes its state from close to open by moving away its closed contacts. Circuitbreaker try to block large amount of fault-current from flowing, and these transients appear in the form of multitude-voltage around circuit-breaker' open contacts. This large amount of voltage is known as transient recovery voltage (T.R.V). This T.R.V is normally three to five-times of the rated operating voltage. Circuit-breaker failure/damage occurs only when it is unable to withstand this T.R.V for which it is designed. In this research, methodology is developed to correctly find the value of T.R.V by taking care of various abnormal and worst-case switching scenarios. T.R.V curtailment is a serious challenge for the power system experts. This study is a competent effort to address this challenge. Electromagnetic-Alternative Transient Program (EM-A.T.P) software is used for simulation of the test network IEEE-39 bus system.
\end{abstract}

Keywords - Abnormal Switching; S.L.G; D.L.G; Circuitbreaker; T.R.V; EM-A.T.P software; IEEE-39 bus system.

\section{INTRODUCTION}

Whenever a circuit changes its operating-state, it generates transients. These transients produce high voltage around the contacts of a circuit-breaker known as transient recovery voltage (T.R.V). T.R.V depends upon circuit-parameters and conditions. T.R.V has a very important role in circuit-breaker, that's why it is very important to carry-out T.V.R study for circuit-breakers. [1]

Normally, circuit-breaker's selection is based upon its short-circuit withstand capability in a circuit. When a fault occurs, circuit-breaker interrupts that fault-current. This interruption of fault-current give rise to T.R.V, which produce maligning impacts on circuit-breaker. Different circuits have different T.R.V values due to different circuit parameters. It has multitude voltage and frequency. T.R.V is graphically represented in figure 1.

"T.R.V is each-step voltage-difference of current-entering and leaving points of a circuit-breaker. In a fault event, incoming-side of circuit-breaker try to maintain nominal voltage and frequency, while outgoing-side faces high voltage and frequency. This voltage difference is called T.R.V."

Whenever there is circuit-breaker operation, there will be T.R.V. But the worst-case T.R.V is produced when circuitbreaker faces fault-current. Hence, circuit-breaker selection must be based upon severity of T.R.V. [1]

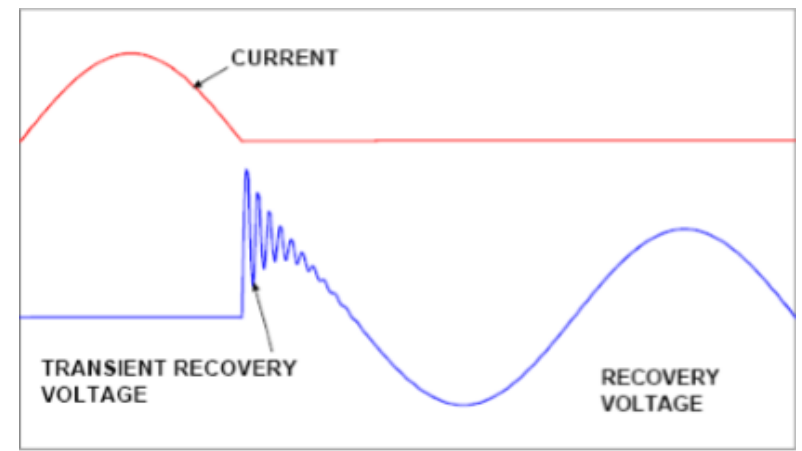

Figure 1: T.R.V

(1) T.R.V impacts on circuit breaker:

$\begin{array}{cl}\text { i. } & \text { Insulation- breakdown } \\ \text { ii. } & \text { Air-breakdown } \\ \text { iii. } & \text { Arcing-Medium-breakdown } \\ \text { iv. } & \text { Arc-Restriking Occurrence }\end{array}$

(2) Impacting factors of T.R.V:

i. System's load-characteristics.

ii. Fault study information of a system.

iii. Circuit-breaker' characteristics.

iv. Transmission-lines characteristics. 


$\begin{aligned} \text { v. } & \text { Internal-characteristics of C.B. } \\ \text { vi. } & \text { Grounding effect. } \\ \text { vii. } & \text { T.R.V types and its wave-shape }\end{aligned}$

(3) Simulation Software:

Electromagnetic-Alternative Transient Program (E.MA.T.P):

This software uses time-domain contingency variables. It collects the data of every circuit-component and converts this data into circuit-equations (differential-equations). The it solves these circuit-equations using integration-laws (Trapezium-rule) and Laplace-transforms. To produce the output in real-world time-domain, it uses inverse-Laplace transforms. [3] [4]

In this software model, electrical network is graphically represented. It is an easy drop-down software environment. It has the ability of multi-tasking, running multiple simulations at a time. All the circuits available in A.T.PDRAW are editable. [3] [4]

(4) Test Network:

New-England IEEE based 39-bus network:

IEEE based 39-bus test-network is a ten machines power system. Considered as widely used test network for research in power systems, it is mostly used in power system stability studies and other power system problems. There are many reasons for using this network as a simulation model instead of using a pragmatic network model, such as: [5]

i. Pragmatic model credentials are comparatively private.

ii. Pragmatic model outcomes are mainly nonspecific and particularly, fewer in number.

iii. Arithmetic scheming is tough since its data is in huge amount.

iv. Software abilities constraints is another drawback of a practical model, hence making it hard to handle huge amount of data.

v. System' data, static as well as dynamic data, is very hard to maintain. [5]

\section{LITERATURE REVIEW}

Transient Recovery Voltage (T.R.V) depends on two categories of factors i.e. internal factors and external factors. These factors are categorized with reference to a circuit breaker of consideration. Internal factors are meant for internal characteristics of a circuit breaker e.g. arc characteristics (arching time, arc voltage, arc current etc.), arc quenching mechanism, and insulation level of circuit breaker. While external factors include type of fault, type of load connected, location of fault, length of transmission line, type of grounding, and surge capacitors. Some past work regarding T.R.V issue is briefed below.

\section{A. Arc designing to cop up with T.R.V issue}

Existence of a fault nearby to a circuit-breaker on the transmission-line is considered as the T.R.V from the transmission-line side. Short length of the transmission-line amid the circuit-breaker and fault-location produced this T.R.V. In the intermediate period of starting-T.R.V on transmission-line side, lasting for comparatively large timespan of some microseconds, SF6 circuit-breaker has the possibility of prevalence of thermal-breakdown. The way for determining T.R.V on the short transmission-line side subject to short-line fault scenario, is done by I.E.C-standards. [6]

T.R.V data that a circuit-breaker possibly to face during service is highly important in its designing and operation. sawtooth wave-shaped T.R.Vs are related to short line fault conditions. The sawtooth-shaped T.R.V's rising rate (ROR) is usually higher than that of encountered with oscillatory-T.R.V $\&$ it is a function of the line's characteristic-impedance. Peak value of T.R.V is normally lesser, due to distributed nature of parameters of transmission-line. Due to these parameters effect, travelling wave-shaped oscillation is observed in transmission-line voltage having positive and negative reflections at the location of fault \& open-contacts of circuitbreaker, respectively. During the.starting portion of the T.R.V only triangular-shaped line side.T.R.V is significant. Fault current is high when fault is in the vicinity of a circuit-breaker, higher will be the initial rising-rate of the T.R.V. As the returning time required.for reflected-wave smaller, the peak of this wave drops. For short-line faults, T.R.V measurement are carried out using only single-phase faults for initial time span of fault. Assuming changes in source voltage is slight . [6]

EM-A.T.P software is used for T.R.V calculations and results comparison is done through I.E.C-standards. arc resistance modelling for SF-6 circuit-breaker is very important as it plays its role in initial-T.R.V. [6]

\section{B. T.R.V's role investigation in circuit breaker failure analysis}

T.R.V investigation studies are required to find out whether the circuit-breaker failure is linked with a T.R.V issue. T.R.V analysis is used to verify whether the circuit-breaker' rating can withstand these T.R.V's. [7]

When connected power system have fault scenarios of T.R.V-characteristics exceeding the rating of circuit-breaker, there is possibility of failing of C.B to interrupt fault-current. After the extinguishing of arc, the voltage producing around the C.B contacts called transient-recovery voltage (T.R.V). Inductive and capacitive parameters connected to the bus determine the behavior and shape of transients. [7]

For successful fault disruption, the fault-interrupting medium voltage known as break-down voltage, must always be higher than the T.R.V. If breaker rating is less than peak value of T.R.V, the increased value of T.R.V around the contact-gap will eventually breakdown the fault intruding medium. In other conditions, if the T.R.V-peak is within safe operating limits of the breaker, there is greater possibility of current re-ignition occurrence as initial micro-seconds just after current disruption the interrupting medium is still conducting and not 
become an insulator; it may have high conductivity and results in conduction. [7]

In starting few micro-seconds if T.R.V rate is high-rising, it may conjoin a current flow sufficient for arc-heating and reestablish the current-conduction. The T.R.V modification can be done by modifying the design of circuit-breaker or by means of added components. The best ever accepted methods are:

1. When the T.R.V is larger than circuit-breaker capacity, interchanging the existing C.B by one of greater voltage category, greater disrupting capacity, or together. As an substitute Zinc. Oxide -devices can also be used to decrease T.R.V. [7]

2. To modify the loading impedance of the circuit with the addition of capacitors, when the rising rate of T.R.V surpasses the definite value. [7]

In past, the focus was mainly upon internal factors of circuit breaker whenever there is need of addressing this issue of Transient Recovery Voltages (T.R.V). In recent developments, it is concluded that internal factors of circuit breaker play very little or no role in T.R.V. Therefore, in this research, my focus is only upon external factors. All of the external factors, upon which T.R.V depend, are designed to measure and preview the value of T.R.V. A bigger test network i.e. IEEE-39 bus system, is selected for simulation of different cases of T.R.V. This research is unique in its nature, as not only T.R.V measurement is presented, but also the mitigation of theses T.R.Vs is also presented.

\section{METHODOLOGY}

Methodology was developed in the following steps:

\section{A. ATP simulation model development of IEEE-39 bus system}

First of all, IEEE-39 bus system' components are modeled to get actual values as ATP software works on actual values rather than per unit values. It includes generator modeling, transmission line modeling, and load modeling.

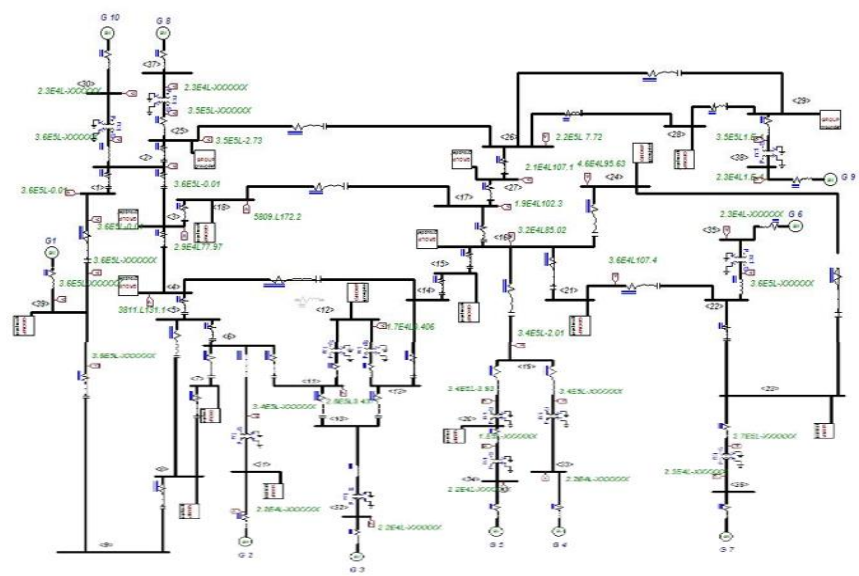

Figure 2: A.T.P Model of 39-bus network

\section{B. Transient equations' derivation using network analysis}

Mathematical representations and circuit analysis techniques are used for different networks to find out their transient equations. RL, RC, and LC networks are analyzed. LC network voltage equation is the actual mathematical representation of T.R.V.

RL circuit analysis:

$$
\mathrm{I}(\mathrm{t})=\frac{\mathrm{Vm}}{\sqrt{R^{2}+L^{2} \omega^{2}}}\left[\operatorname{Sin}(\omega t+\theta-\phi)-\operatorname{Sin}(\theta-\phi) e^{-\alpha t}\right]
$$

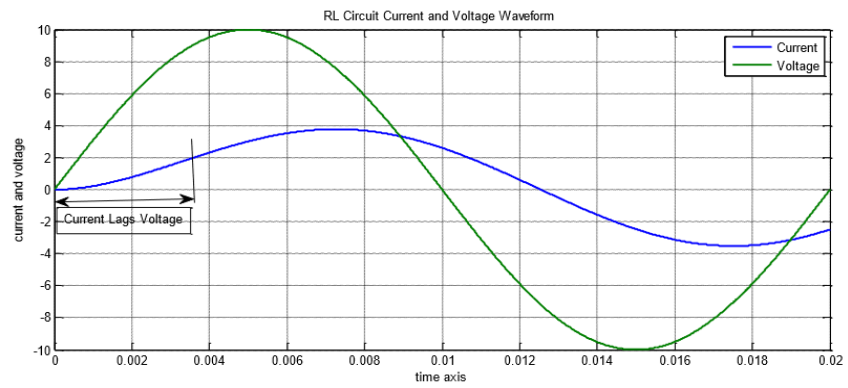

Figure 3: RL circuit waveform

RC circuit analysis:

$$
\mathrm{V}_{\mathrm{c}}(\mathrm{t})=\mathrm{V}_{\mathrm{A}}\left[\operatorname{Sin}(\omega t+\theta-\phi)-\operatorname{Sin}(\theta-\phi) e^{-\alpha t}\right]
$$

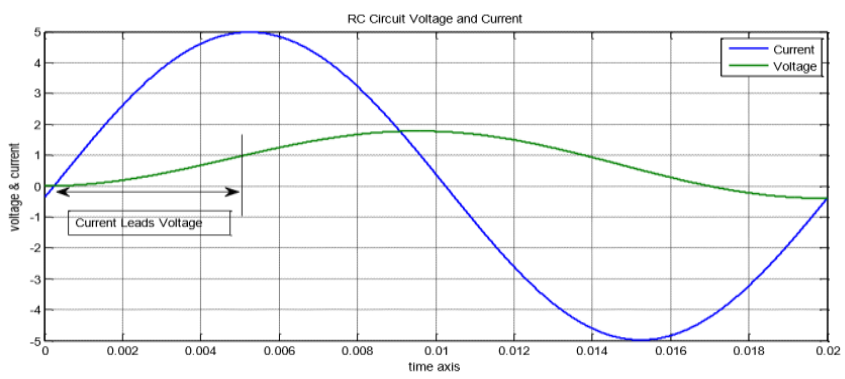

Figure 4: RC circuit waveform

LC circuit analysis:

$$
\mathrm{Vc}(\mathrm{t})=\mathrm{Vm}\left(1-\operatorname{Cos} \omega_{\mathrm{o}} t\right)
$$

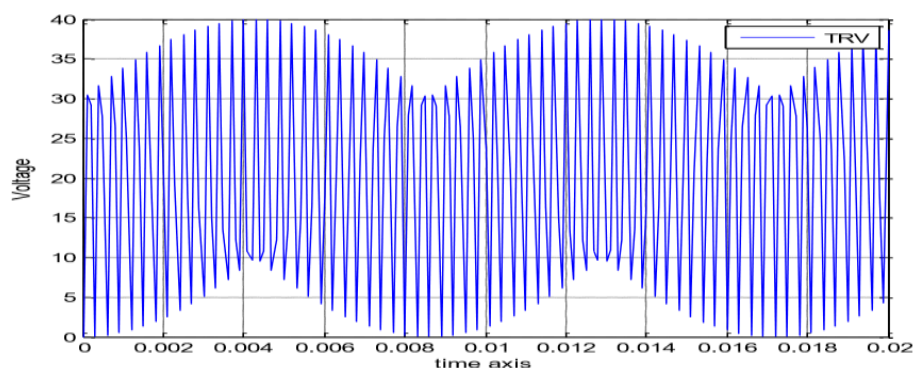

Figure 5: LC circuit waveform

\section{Simulation of external factors affecting T.R.V in ATP}

External factors include nature of fault, load-characteristics, transmission-line length, characteristic-impedance, faultlocation, and grounding effect. [8] [9] 


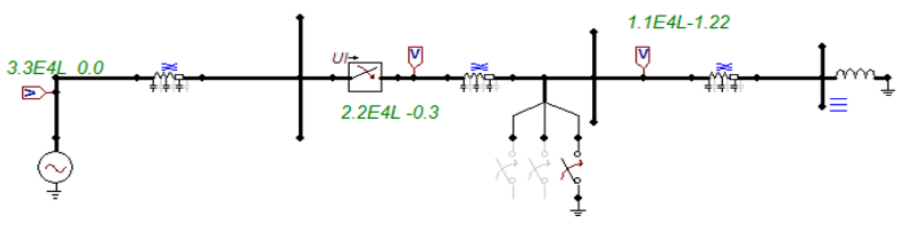

Figure 6: Test Network

1. Load Characteristics' Impact on T.R.V

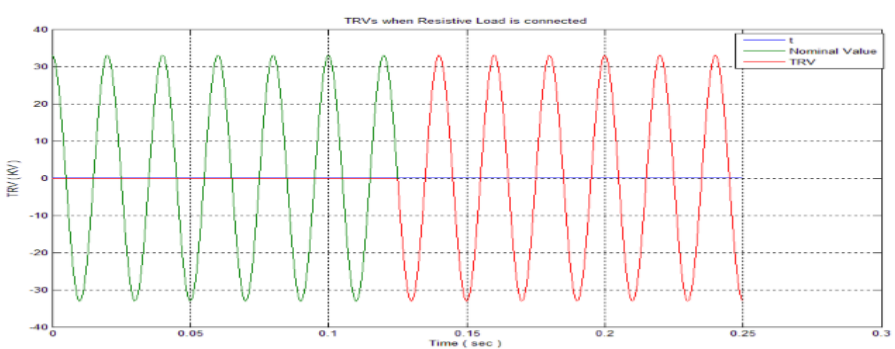

Figure 7: Resistive Load

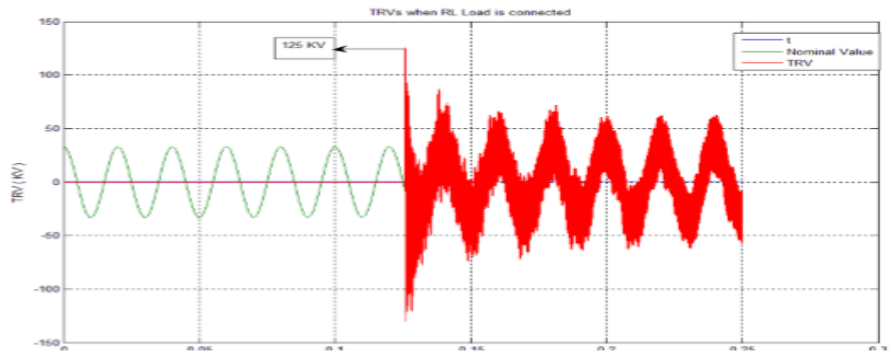

Figure 8: RL Load

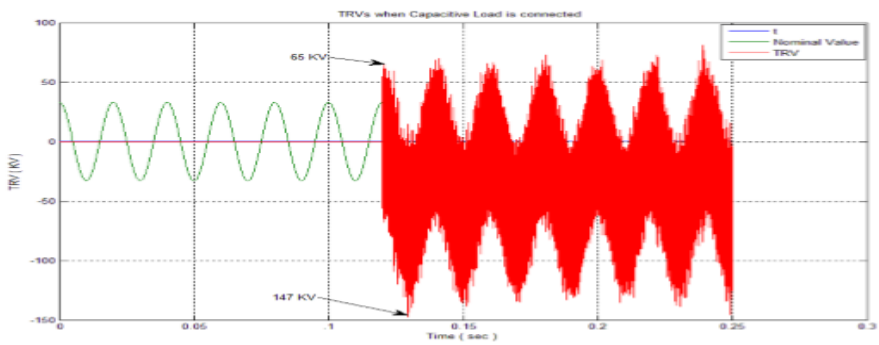

Figure 9: Capacitive Load

2. Transmission-line length [9]

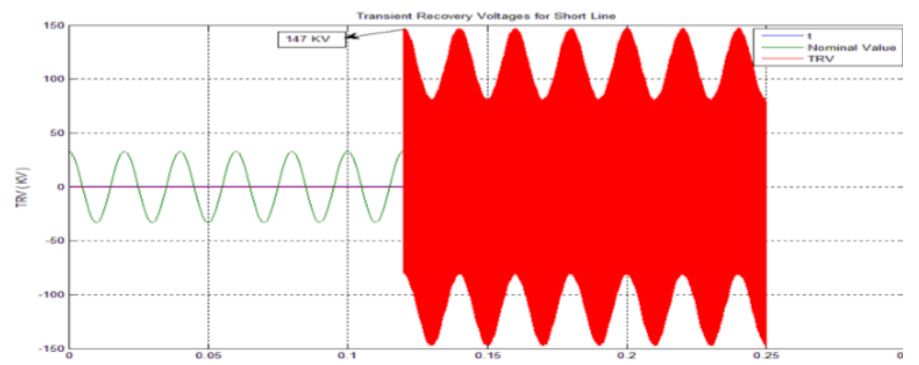

Figure 10: Short Line $(80 \mathrm{kM})$

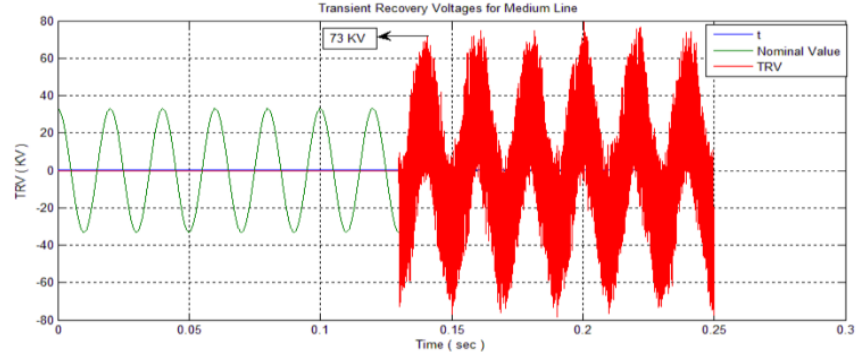

Figure 11: Medium Line (250 kM)

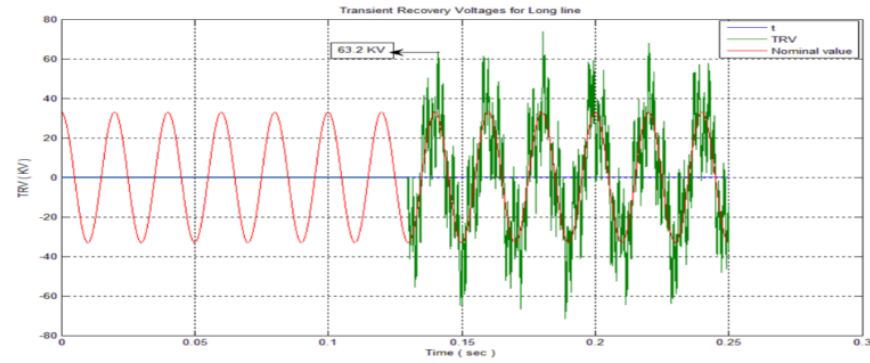

Figure 12: Long Line (>250 kM)

3. Fault location

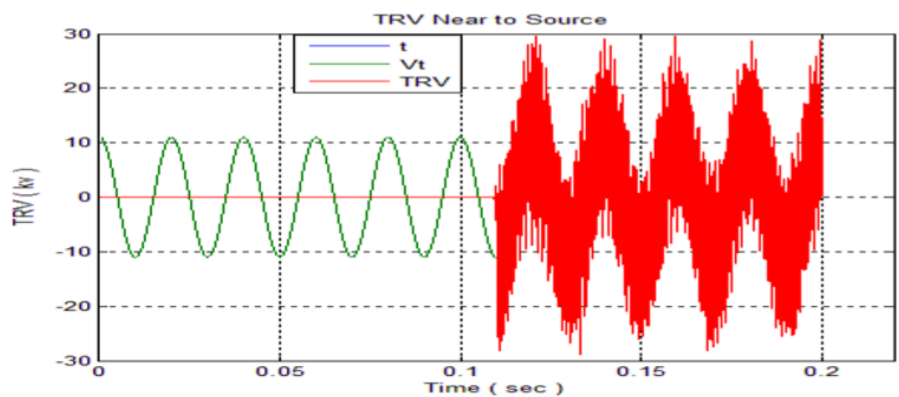

Figure 13: Fault near to source

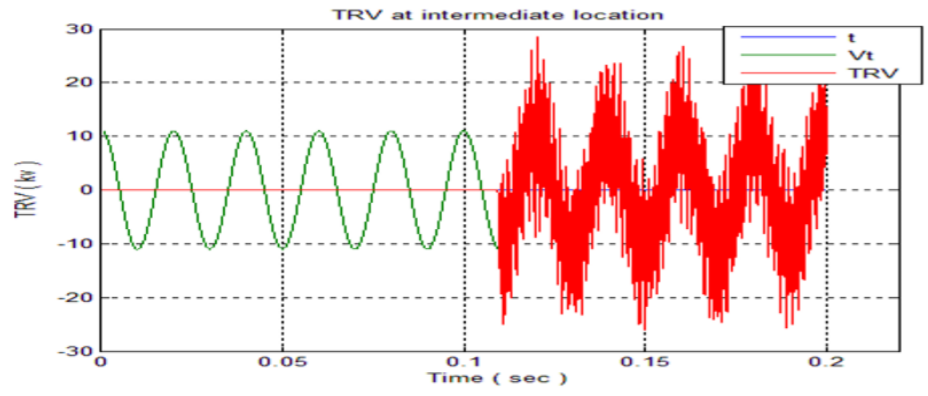

Figure 14: Fault at intermediate location 


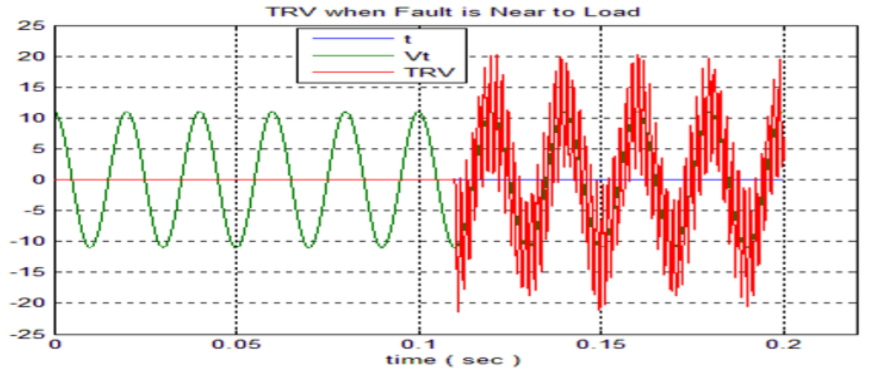

Figure 15: Fault near to load

\section{Type of Grounding}

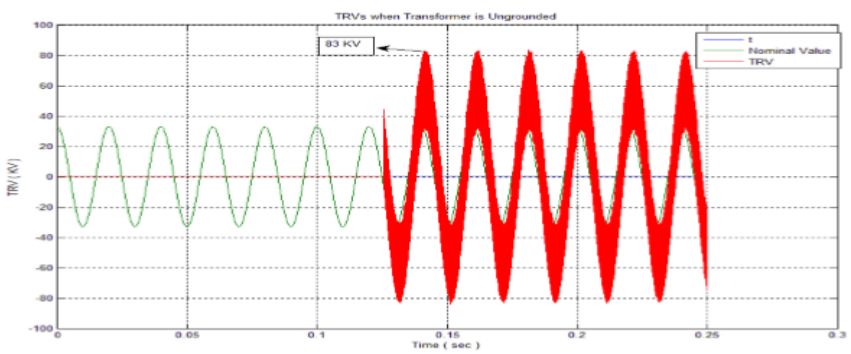

Figure 16: Ungrounded System

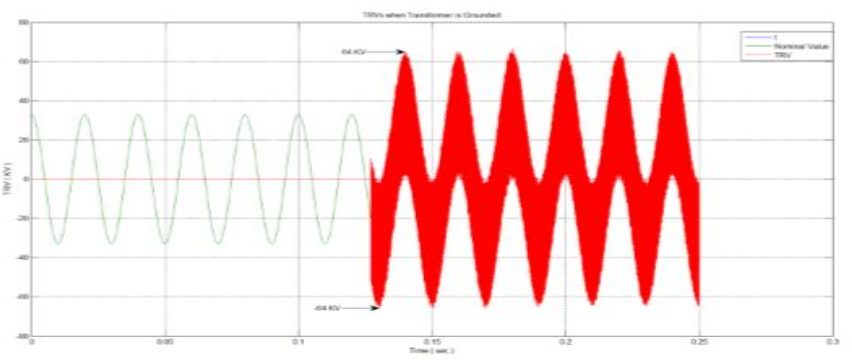

Figure 17: Grounded System

5. Characteristic Impedance $\mathrm{Zo}=\operatorname{sqrt}(\mathrm{L} / \mathrm{C})$

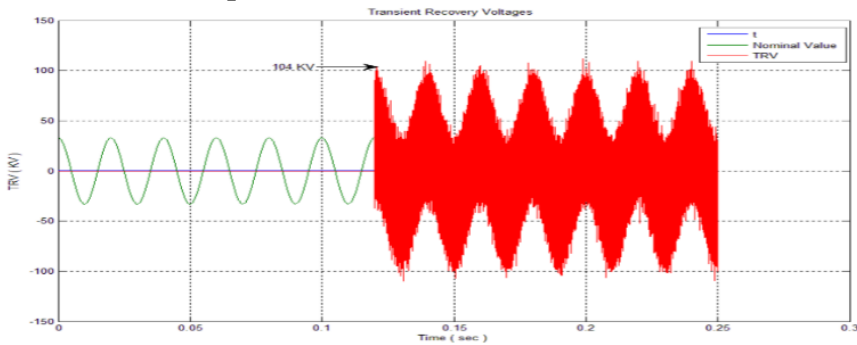

Figure 18: Case\#1: $\mathrm{L}=1.5 \mathrm{mH}, \mathrm{C}=0.1 \mathrm{uF}$

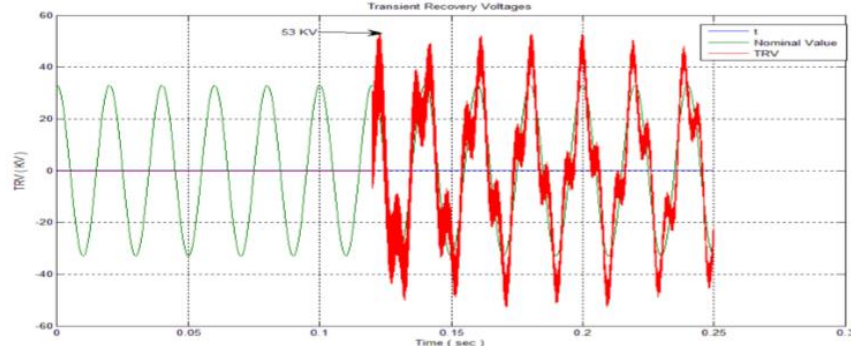

Figure 19: Case\#2: $\mathrm{L}=15 \mathrm{mH}, \mathrm{C}=10 \mathrm{uF}$

Table 1: A.T.P Simulation Results of External Factors

\begin{tabular}{|c|c|c|}
\hline External Factors & $\begin{array}{l}\text { Operating } \\
\text { Voltage }\end{array}$ & Measured T.R.V \\
\hline Load-Characteristics & $33-\mathrm{kV}$ & $\begin{array}{c}\text { 33-kV ( R ) } \\
104-k V(\text { L }) \\
105-k V(\text { C }) \\
125-k V(\text { R.L })\end{array}$ \\
\hline $\begin{array}{l}\text { Transmission-line } \\
\text { length }\end{array}$ & $33-\mathrm{kV}$ & $\begin{array}{c}\text { 147-kV ( Short ) } \\
\text { 73-kV ( Medium ) } \\
\text { 63-kV ( Long ) }\end{array}$ \\
\hline $\begin{array}{l}\text { Characteristic- } \\
\text { impedance }\end{array}$ & $33-\mathrm{kV}$ & $\begin{array}{l}106-\mathrm{kV}(\mathrm{L}=1.5 \mathrm{mH}, \mathrm{C}=0.1 \mu \mathrm{F}) \\
53-\mathrm{kV}(\mathrm{L}=15 \mathrm{mH}, \mathrm{C}=10 \mu \mathrm{F})\end{array}$ \\
\hline Fault-location & $11-\mathrm{kV}$ & $\begin{array}{c}\text { 32-kV ( Source Side }) \\
\text { 26-kV ( Midway ) } \\
\text { 22-kV ( Load Side ) }\end{array}$ \\
\hline Grounding effect & $33-\mathrm{kV}$ & $\begin{array}{c}\text { 82-kV ( Un-grounded N/W ) } \\
61-\mathrm{kV} \text { ( Grounded N/W ) }\end{array}$ \\
\hline
\end{tabular}

\section{T.R.V Reduction Techniques}

T.R.V is a quick and multitude phenomenon. Its implications to the power-system and its components are very severe. Following are some of the reasons explaining why this notorious phenomenon must be controlled.

Insulation- breakdown: Circuit-breaker' insulation is under so much stress due to this T.R.V, which causes Insulationbreakdown.

Air-breakdown: T.R.V puts huge stress on the air present in the space surrounding circuit-breaker, in close-proximity of fault-interrupting circuitry. And ultimately results in airbreakdown.

Arcing-Medium-breakdown: whenever there is a fault, circuit-breaker operates by switching its contacts from close to open state. During this contacts-switching process, arc is produced in the fault-interrupting medium due to faultinterruption. Fault will be successfully interrupted if this arcmedium sustains large value T.R.V. If arc-medium fails to sustain T.R.V, arc-medium collapses. As a result, current passes continuously through the circuit-breaker even if its contacts are opened. Eventually, circuit-breaker burns-down.

Taking in consideration the above mentioned reasons about severity of T.R.V, it is inevitable to devise techniques for curtailment of T.R.V. [2] [10] 


\section{Switching-Resistance technique}

T.R.V can be controlled by correctly managing the arcresistance. In this technique, rather than allowing the current to pass through the imaginary-resistance of the arc established, a physical by-pass resistor is employed around the contacts. So, current flow is divided between these two resistances. As a result, current flowing through arc decreases and deionization process of arc speeds-up. This speeding-up of the deionization process quickly quenches the arc. Now, the resistance of the arc is increased. Lesser the current in the arc-path, quickly the arc will die-out. When the arc fully dies-out, circuit-breaker is successful in interrupting the fault.

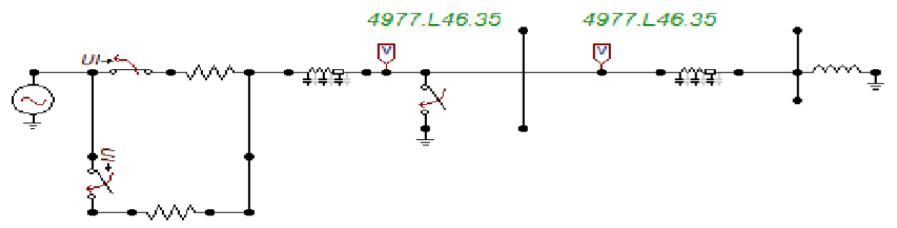

Figure 20: Switching-Resistance circuit

For $11-\mathrm{kV}$ test network, switching-resistance technique result given below:

Measured T.R.V (without curtailment $)=21.5-\mathrm{kV}$

T.R.V reduction (after curtailment) $=11.5-\mathrm{kV}$

Following are the A.T.P simulation results of above mentioned circuit.

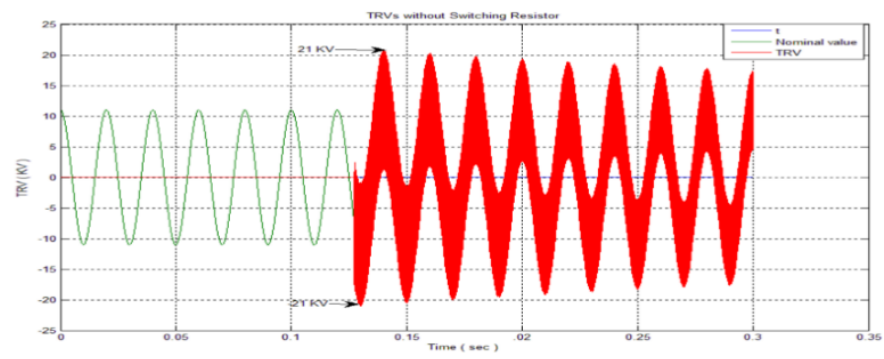

Figure 21: T.R.V without switching resistor

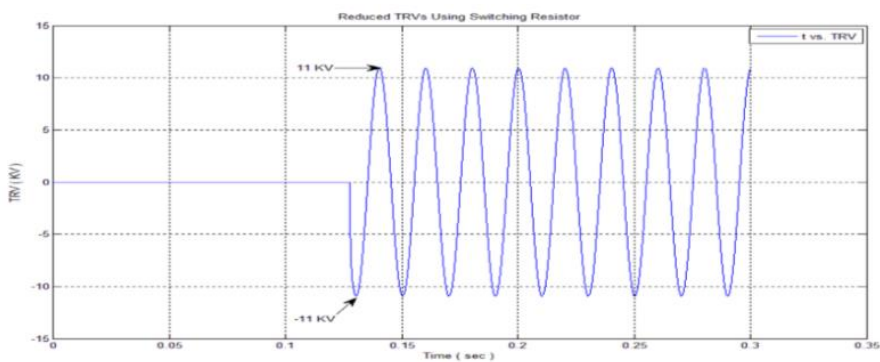

Figure 22: T.R.V with switching resistor

It is concluded that T.R.V can be significantly reduced (about $45 \%$ ) by using switching-resistor.

\section{Surge-absorbing Capacitor Switching}

In this technique, voltage-surge absorber is used, which is basically a capacitor. This capacitor offers impedance to the fault-current.

$$
\mathrm{X}_{\mathrm{C}}=\frac{1}{\mathrm{j} \omega \mathrm{C}}
$$

This capacitor absorbs surges accompanying with voltage. A small-value capacitor is used to offer large impedance to the fault-current. Surges are termed as high-voltage and highfrequency transients. As frequency is very large, its capacitiveimpedance $\left(X_{C}\right)$ becomes very small. And high-frequency voltage surges are diverted to ground-path. In this way, our circuitry is protected from high-voltage surges. Fault-current absorption by this capacitor depends upon its size.

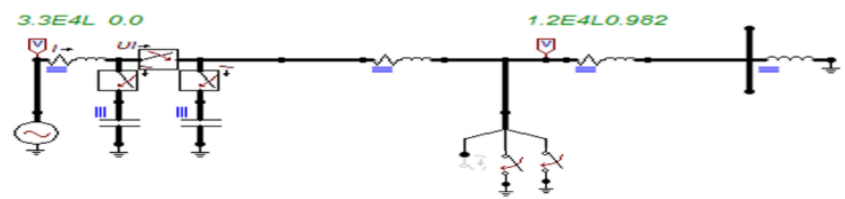

Figure 23: Surge-absorbing capacitor circuit

For $33-\mathrm{kV}$ test network, switching-capacitance technique result given below:

Measured T.R.V (without curtailment) $=68.6-\mathrm{kV}$

T.R.V reduction (after curtailment $)=48-\mathrm{kV}$

Following are the A.T.P simulation results of above mentioned circuit.

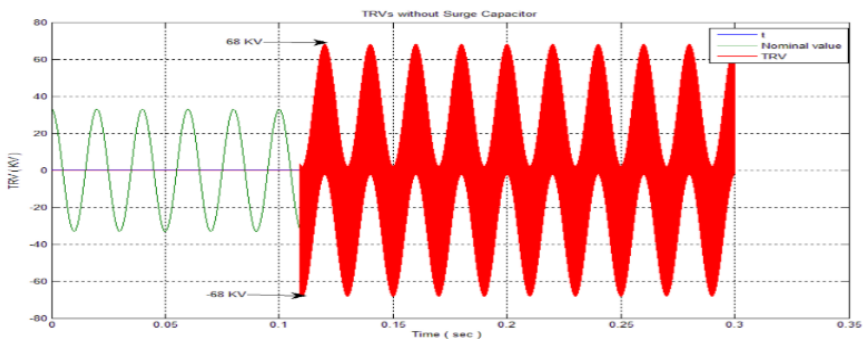

Figure 24: T.R.V without surge-absorbing capacitor

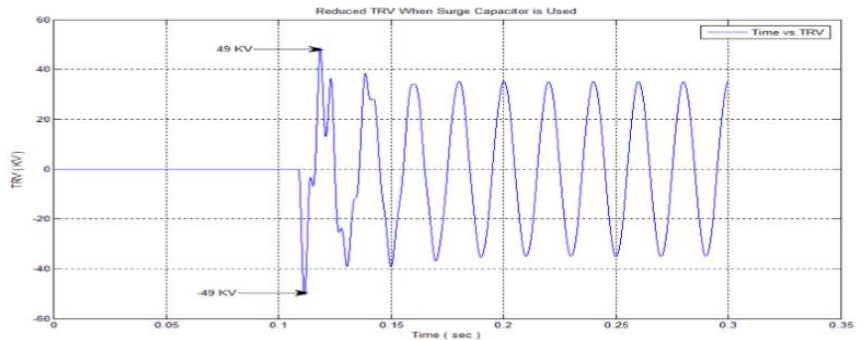

Figure 25: T.R.V with surge-absorbing capacitor

It is concluded that T.R.V can be significantly reduced (about $35 \%$ ) by using surge-absorbing capacitor. 
IV. RESULTS \& DISCUSSIONS

A. T.R.V Simulations of IEEE-39 bus system

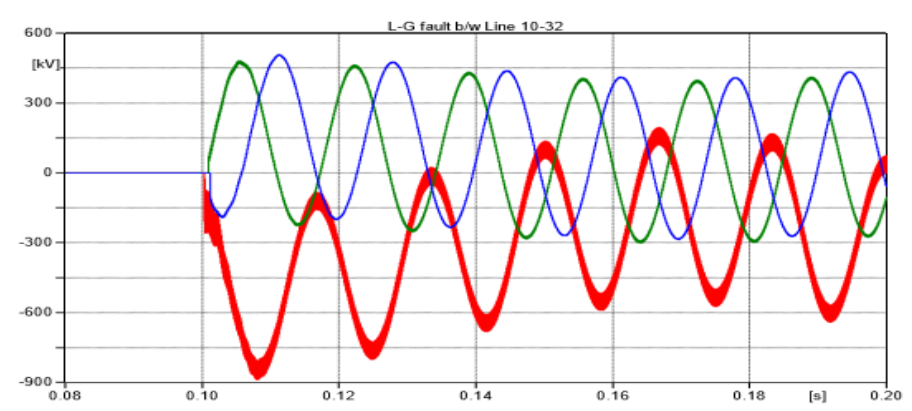

Figure 26: T.R.V for S-L-G fault at buses $10 \& 32$

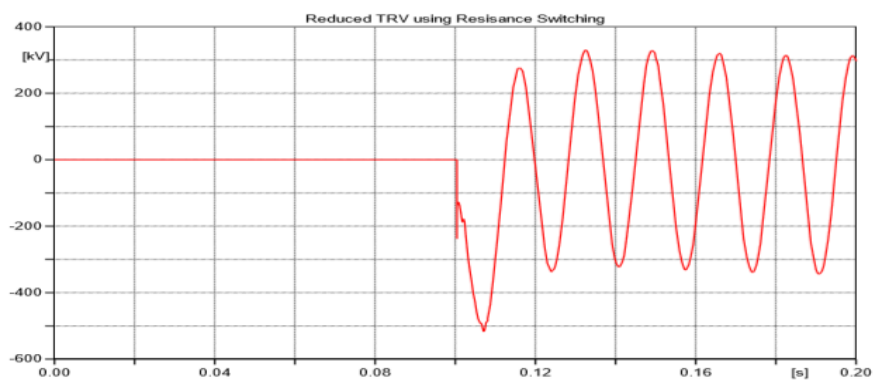

Figure 27: Reduced T.R.V for S-L-G fault at buses $10 \& 32$

Operating Voltage $=345-\mathrm{kV}$

Measured T.R.V $=880-\mathrm{kV}$

Measured T.R.V in an S.L.G-fault case is 2.8-times of operating voltage.

T.R.V-Reduction (switching-resistance technique) $=510 \mathrm{kV}$

In this case, we find $43 \%\left(\frac{900-510}{900} \times 100\right)$ reduction in T.R.V.

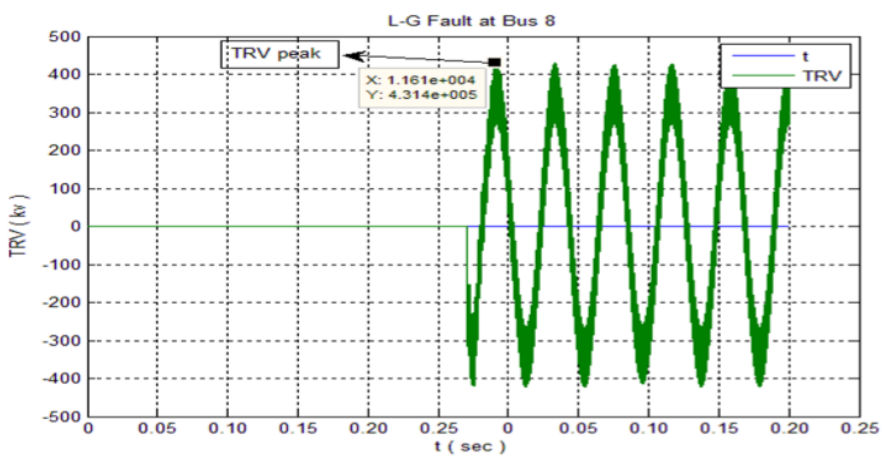

Figure 28: T.R.V for S-L-G fault at buse 8

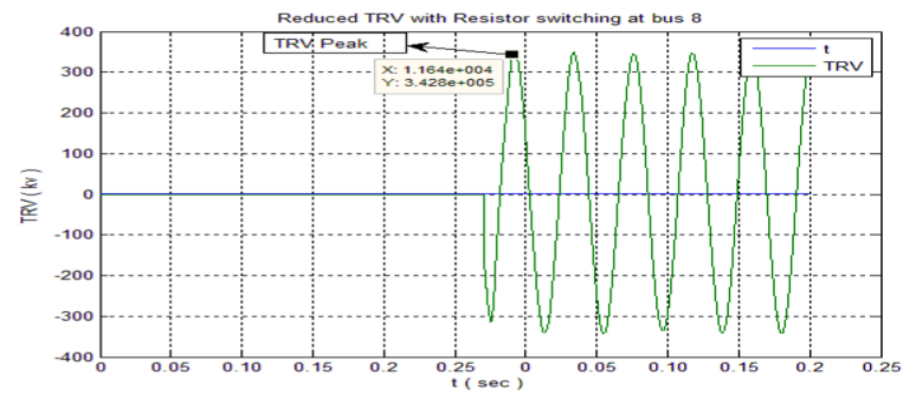

Figure 29: Reduced T.R.V for S-L-G fault at buse 8

Operating Voltage $=345-\mathrm{kV}$

Measured T.R.V for Case- $1=452-\mathrm{kV}$

T.R.V-Reduction (switching-resistance technique) $=357 \mathrm{kV}$

In this case, we achieved $21 \%\left(\frac{452-357}{452} \times 100\right)$ reduction in T.R.V.

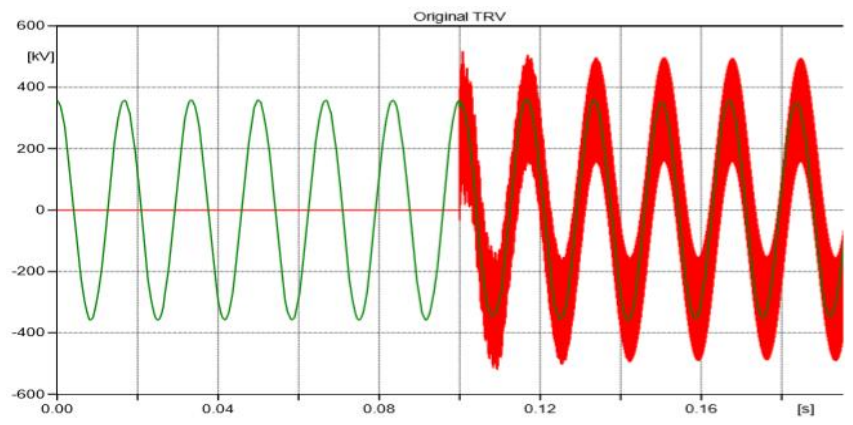

Figure 30: T.R.V for S-L-G fault at buses 6-31

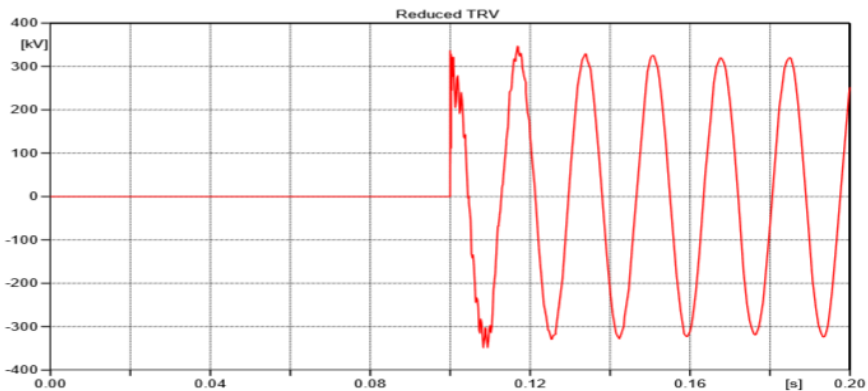

Figure 31: Reduced T.R.V for S-L-G fault at buse 6-31

Operating Voltage $=345-\mathrm{kV}$

Measured T.R.V for Case- $1=570-\mathrm{kV}$

T.R.V-Reduction (switching-resistance technique) $=360 \mathrm{kV}$

In this case, we achieved $37 \%\left(\frac{570-360}{570} \times 100\right)$ reduction in T.R.V.

\section{CONCLUSION}

Transient Recovery Voltages (T.R.V) depends on two types of factors i.e. internal factors and external factors. Internal factors include arc characteristics, arc quenching mechanism, and insulation level of circuit breaker. While external factors include fault type, type of load connected, location of fault, 
transmission-line length, grounding type, and surge capacitors. In this research, T.R.V is measured and previewed using external factors of circuit breaker. It was observed that T.R.V is highest for R.L load, short transmission line, ungrounded network, large surge impedance, and fault location near source side. Two techniques are used to mitigate these multitude T.R.V that is switching resistance technique and switching capacitance technique. Both of these techniques work in support of reducing fault-current, which ultimately reduces the T.R.V. Switching resistance technique achieved $45 \%$ reduction in T.R.V while switching capacitance technique achieved $35 \%$ reduction in T.R.V. This reduction in T.R.V is fair enough for circuit breaker to operate within limits and prevent the circuit breaker from failing due to large value T.R.V. In the last phase of this research, T.R.V measurements of IEEE-39 bus test network were performed using different external factors that affect T.R.V. These measurements were then cross-checked for validation using formulas for T.R.V. and reduction of the same measurements was performed by using resistance switching and capacitance switching technique. T.R.V was significantly reduced for about $35 \%$.

\section{REFERENCES}

11 D. Dufournet and .. Alexander, "Transient Recovery (TRV) for High Voltage Circuit Breakers".

IEC , "High-voltage switchgear and controlgear", High-

[2] voltage alternating-current circuit-breakers,," in IEC 62271100: , 2003.

[3] "https://www.atpdraw.net," [Online].

[4] "www.ece.mtu.edu>atp," [Online].

[5] "http://psdyn.ece.wisc.edu/IEEE_benchmarks," [Online].

[6] A. Greenwood, "Electrical Transients in Power Systems,," Wiley, 1991.

P. W. J. Z. D. M. D. K. K.-W. L. Xuegong Wang, "Transient

[7] Recovery Voltage Investigation in the Application of $15 \mathrm{kV}$ Circuit Breaker Failure," Vols. Paper No. IPST05 - 224, IPST'05.

A. A. B. S. S. Chandankar, "Review: To Investigate Impacts of Various Factors on the Characteristics of Transient

[8] Recovery Voltage.,", International Journal of Advanced Research in Electrical, Electronics and Instrumentation Engineering, vol. 5.

I. U. D. F.-G. Božidar Filipoviü-Grpiü, "Analysis of Transient

[9] Recovery Voltage in $400 \mathrm{kV}$ SF6 Circuit Breaker Due to

[9] Transmission Line Faults," Vols. Vol. 6, N. 5, SeptemberOctober 2011.

R. Colclaser, "The Transient Recovery Voltage Application of

10] Power Circuit Breakers," IEEE Transactions on Power Apparatus and Systems, Vols. PAS-91, no. 5, pp.1941-1947,, Sept. 1972.

\section{Rahman Ullah Khan}

The author has received BE degree in Electrical Engineering from NED University of Engineering \& Technology, Karachi in 2014. He has one year industry experience of Fauji Fertilizer Bin-Qasim Limited (FFBL) from 2014 to 2015. Currently, he is doing MS in Electrical Energy Systems Engineering (EESE) from U.S.-Pakistan Center for Advanced Studies in-Energy (USPCAS-E), University of Engineering \& Technology, Peshawar. He has availed exchange program to the partner university of USPCAS-E Arizona State University (ASU), USA in 2017, as a research scholar. He has good hands on experience in the field of electrical power and renewable energies especially PV reliability. 